$\Rightarrow$ LIVER CANCER

NAFLD-HCC: target cholesterol

Squalene epoxidase (SQLE), an enzyme involved in cholesterol biosynthesis, has a role in the development of hepatocellular carcinoma associated with nonalcoholic fatty liver disease (NAFLD-HCC), according to new research. The study also demonstrates that terbinafine, an approved antifungal agent that targets SQLE, inhibits NAFLD$\mathrm{HCC}$ in cellular and animal models, highlighting its therapeutic potential as a repurposed drug.

With the increasing levels of obesity worldwide, fatty liver disease is becoming a major health problem and an emerging risk factor for liver cancer. Jun Yu and colleagues investigated the molecular mechanisms that underlie NAFLD-HCC, using transcriptome analysis to identify potential gene candidates.

RNA sequencing analysis revealed that SQLE was overexpressed in human NAFLD-HCC tissue and in obesity-associated NAFLD-HCC mouse models. Moreover, SQLE expression was associated with poor survival in patients with hepatocellular carcinoma and confirmed as an independent prognostic factor.

SQLE acted as an oncogene in the context of NAFLD-HCC. SQLE promoted cell growth and inhibited apoptosis in NAFLD-HCC cell lines, and transgenic expression of SQLE specifically in hepatocytes accelerated tumour growth in NAFLD-HCC mouse models. Examining the mechanism of action, the researchers found that SQLE promoted tumorigenesis via regulation of cholesteryl esters and reactive oxygen species. Crucially, pharmacological inhibition of SQLE with terbinafine suppressed NAFLD-HCC growth in vitro and in vivo.

"Epidemiological and preclinical studies have suggested a causative role of cholesterol and its metabolites in cancer development," explains Yu. "While the majority of published studies focused on HMG-CoA reductase as it is the first rate-limiting enzyme, the role of SQLE on cholesterol homeostasis was frequently ignored." The researchers plan to optimize the use of terbinafine for the prevention and treatment of NAFLD-HCC, and ultimately hope to translate their experimental work to the clinic.

Katrina Ray

ORIGINAL ARTICLE Liu, D. et al. Squalene epoxidase drives NAFLD-induced hepatocellular carcinoma and is a pharmaceutical target. Sci. Transl Med. 10, eaap9840 (2018)

$\Rightarrow$ BILIARY TRACT

\title{
De novo hepatocyte-derived biliary system
}

In a demonstration of transdifferentiation, a new study published in Nature shows that hepatocytes in mice can convert to cholangiocytes to build a new functioning biliary system that was absent in development. By elucidating the driver of this mechanism, these findings redefine hepatocyte plasticity and could lead to novel treatments for cholestatic liver diseases.

Previous work by the authors had led to the generation of NOTCH-deficient mice born without intrahepatic bile ducts (IHBD), mimicking a phenotype seen in patients with Alagille syndrome. However, formation of IHBDs was later observed in these mice, prompting an investigation into the cellular origin of the new biliary system using genetic cell fate tracing. "We developed new tools based on the Flp recombinase system to specifically label hepatocytes in the mouse liver," explains author Holger Willenbring. "We found that hepatocytes can become cholangiocytes that are indistinguishable from primary cholangiocytes.”

Changing cellular identity to replenish missing cells, known as transdifferentiation, has been observed in various other tissues. However, in the latest study, the hepatocyte-derived cholangiocytes formed functional IHBDs without guidance from pre-existing bile ducts. Such de novo formation of mammalian structures from transdifferentiation was previously unknown.

"We also identified the molecular mechanism driving this process," reports author Stacey Huppert. "In contrast to bile duct development, where NOTCH signalling is essential, we found that TGF $\beta$ signalling can compensate in the adult liver." Furthermore, TGF $\beta$ signalling was active in IHBDs found in some patients with Alagille syndrome and could be targeted to enhance the formation of the biliary system from hepatocytes in mice.

"We are now using the understanding and tools generated in our study to develop hepatocyte-transdifferentiation-based strategies for therapy of cholestatic liver diseases," concludes Willenbring.

Iain Dickson

ORIGINAL ARTICLE Schaub, J. R. et al. De novo formation of the biliary system by TGF $\beta$-mediated hepatocyte transdifferentiation. Nature 557, 247-251 (2018)

\section{INFECTIOUS DISEASE}

\section{Tuft cells revealed as norovirus target}

Tuft cells are the targets of norovirus infection in mice, according to a new study published in Science. The findings have important implications for understanding norovirus pathogenesis and how interactions with other gut organisms alter the disease.

Globally, human noroviruses are the leading cause of acute viral gastroenteritis, an important disease burden responsible for around 200,000 deaths a year. There are no vaccines or antiviral therapies for human noroviruses, in part because understanding of the mechanisms of norovirus pathogenesis is limited.

In a new study, Craig Wilen and colleagues sought to shed light on how mouse noroviruses interact with the host and cause disease. "We use mouse norovirus as a model for human norovirus because mouse norovirus can replicate in cell culture and has a reverse genetics system," says Wilen.

As the cellular tropism of norovirus in mice or humans was unclear, the researchers first used bone marrow transplants in a mouse model of norovirus infection to show that the target cell was radiation-resistant, and therefore an epithelial cell.
Immunofluorescence microscopy of the gut epithelium in these mice then revealed that tuft cells were the exclusive target of infection, a finding confirmed by flow cytometry. "Tuft cells are a very rare chemosensory cell in the lining of the gut that sense intestinal worms and parasites and then initiate an immune response," explains Wilen. "It was quite surprising how so few infected cells $(\sim 100)$ per mouse could cause such substantial infection." Interestingly, tuft cell tropism might link helminth infection and the gut microbiota, which increase tuft cell numbers in the ileum and colon, respectively, to more severe norovirus infection in humans. "This finding may explain why there is greater human norovirus disease in regions that also have higher worm and parasite burdens," Wilen remarks. Future research will aim to clarify how these findings translate to human norovirus infection.

Hugh Thomas

ORIGINAL ARTICLE Wilen, C. B. et al. Tropism for tuft cells determines immune promotion of norovirus pathogenesis. Science 360, 204-208 (2018) 